Zbl Arbeitsmed 2014 $\cdot 64: 7$

DOI 10.1007/s40664-013-0018-9

Online publiziert: 6. Februar 2014

๑) Springer-Verlag Berlin Heidelberg 2014

Redaktion $^{1} \cdot$ D. Groneberg ${ }^{2} \cdot$ A. Luttmann ${ }^{3}$

${ }^{1}$ Springer-Verlag Heidelberg

${ }^{2}$ Institut für Arbeitsmedizin,Sozialmedizin und Umweltmedizin, Goethe-Universität, Frankfurt am Main

${ }^{3}$ IfADo - Leibniz-Institut für Arbeitsforschung, TU Dortmund

\title{
Willkommen bei Springer
} einzureichen. Darüber hinaus wünschen wir uns zahlreiche Artikel neuer Autoren, die hoffentlich Interesse am Zentralblatt für Arbeitsmedizin in neuem Gewande finden mögen.

\section{Schneller publizieren durch Online-First}

Allen Verfassern versprechen wir wie bisher ein qualitätsorientiertes und anspruchsvolles Peer-Review-Verfahren. Zudem werden alle Beiträge zukünftig auch Online-First publiziert und sind damit über den DOI (digital object identifier) bereits vor der Drucklegung im Heft zitierfähig.

\section{Vielfältiges Online-Angebot}

Das Zentralblatt hat nun auch online ein neues Zuhause gefunden. Über www. ZentralblattfuerArbeitsmedizin.de gelangen Sie jetzt zur Homepage Ihrer Zeitschrift auf der Sie alle wichtigen Informationen rund um die Zeitschrift finden u. a. Zielsetzung der Zeitschrift, Herausgeberboard, Hinweise für Autoren, Kon- takte und das Volltextarchiv. Im Volltextarchiv auf SpringerLink, der großen Literaturdatenbank von Springer, stehen Ihnen alle elektronischen Ausgaben Ihrer Zeitschrift zur Verfügung. Die einzelnen Beiträge sind als PDF-Datei im Layout der gedruckten Ausgabe und als HTMLVersion verfügbar. In der HTML-Version können Sie die Vorteile der verlinkten Literatur nutzen und direkt zu den zitierten Quellen gelangen.

\section{》) Volltextarchiv unter www. ZentralblattfuerArbeitsmedizin.de}

》) Fragebögen der CME-Beiträge jetzt online bearbeiten

Die Fragebögen der Weiterbildungsbeiträge können Sie online bearbeiten. Ihre Punkte werden automatisch an die Landesärztekammer übermittelt. Darüber hinaus erhalten Sie ein persönliches Konto in dem Ihre Teilnahmebescheinigungen archiviert werden.

Die vorliegende Ausgabe enthält eine anregende Mischung aus fachwissenschaftlichen Themen, Forschungsbeiträgen und Diskussionen aus dem breiten Spektrum der Arbeitsmedizin. Allen Leserinnen und Lesern eine anregende Lektüre!

Wir wünschen Ihnen viel Spaß beim Lesen des Zentralblatts für Arbeitsmedizin und bitten Sie schon jetzt um jede Form von Lob und Kritik, Anmerkungen und Verbesserungsvorschlägen. Wir freuen uns auf Ihre Reaktion! demie, in der Sie die Fragebögen der Weiterbildungsbeiträge bearbeiten können.

\section{CME als wichtiger Bestandteil}

Die bewährte Rubrik „CME Zertifizierte Fortbildung" wird als fester Bestandteil der Zeitschrift in jeder Ausgabe fortgeführt. Alle Weiterbildungsbeiträge werden von den Landesärztekammern standardmäßig mit 3 Punkten zertifiziert. Mit bis zu 18 Punkten jährlich sieht sich das Zentralblatt für Arbeitsmedizin somit auch als Fortbildungsorgan, und hat den Ehrgeiz sich in diesem Bereich zu verbessern.

\section{Für den Verlag}

Monika Kretz (Leitung Facharztzeitschriften), Dr. Friederike Fellenberg (Redaktionsleitung) und Helena Weber (Redaktion)

Für die Herausgeber

Prof. Dr. David Groneberg (federführender Schriftleiter Medizin), Prof. Dr. Alwin Luttmann (federführender Schriftleiter Technik) 